\title{
Performance of Shannon-entropy compacted $N$-electron wave functions for configuration interaction methods
}

\author{
Diego R. Alcoba ${ }^{1,2} \cdot$ Alicia Torre $^{3}\left(\mathbb{D} \cdot\right.$ Luis Lain $^{3}\left({ }^{\circledR} \cdot\right.$ Gustavo E. Massaccesi $^{4}$ • \\ Ofelia B. Oña ${ }^{5} \cdot$ Paul W. Ayers ${ }^{6} \oplus$ - Mario Van Raemdonck ${ }^{7} \cdot$ Patrick Bultinck $^{7}$ (1) \\ Dimitri Van Neck ${ }^{8}$ (อ)
}

Received: 26 January 2016 / Accepted: 2 May 2016

(C) Springer-Verlag Berlin Heidelberg 2016

\begin{abstract}
The coefficients of full configuration interaction wave functions (FCI) for $N$-electron systems expanded in $N$-electron Slater determinants depend on the orthonormal one-particle basis chosen although the total energy remains invariant. Some bases result in more compact wave functions, i.e. result in fewer determinants with significant expansion coefficients. In this work, the Shannon entropy, as a measure of information content, is evaluated for such wave functions to examine whether there is a relationship between the FCI Shannon entropy of a given basis and the performance of that basis in truncated CI approaches. The results obtained for a set of randomly picked bases are compared to those obtained using the traditional canonical molecular orbitals, natural orbitals, seniority minimising orbitals and a basis that derives from direct minimisation of the Shannon entropy. FCI calculations for selected atomic and molecular systems clearly reflect the influence of the chosen basis. However, it is found that there is no direct relationship between the entropy computed for each basis and truncated CI energies.
\end{abstract}

Patrick Bultinck

Patrick.Bultinck@UGent.be

1 Departamento de Física, Facultad de Ciencias Exactas

y Naturales, Universidad de Buenos Aires, Ciudad

Universitaria, 1428 Buenos Aires, Argentina

2 Instituto de Física de Buenos Aires, Consejo Nacional de Investigaciones Científicas y Técnicas, Ciudad Universitaria, 1428 Buenos Aires, Argentina

3 Departamento de Química Física, Facultad de Ciencia y Tecnología, Universidad del País Vasco, Apdo. 644, 48080 Bilbao, Spain

4 Departamento de Ciencias Exactas, Ciclo Básico Común, Universidad de Buenos Aires, Ciudad Universitaria, 1428 Buenos Aires, Argentina
Keywords Entropy $\cdot$ Configuration interaction · Seniority · DOCI

\section{Introduction}

The full configuration interaction (FCI) wave function, usually expressed in terms of $\mathrm{N}$-electron Slater determinants, corresponds to the exact solution of the Schrödinger equation of an $N$-electron system for a given one-electron basis set. However, its determination comes at a prohibitive computational cost, except for systems with few electrons and for small basis sets. This drawback is overcome by using approximate methods. The FCI wave function expansion includes all possible $\mathrm{N}$-electron determinants that can be obtained from an orthonormal set of orbitals. Hence, the energy associated with the FCI wave function is independent of the actual chosen orthonormal basis. Truncated configuration interaction (CI) methods mitigate the prohibitive computational cost of FCI methods by restricting

Instituto de Investigaciones Fisicoquímicas Teóricas y Aplicadas, Universidad Nacional de la Plata, CCT La Plata, Consejo Nacional de Investigaciones Científicas y Técnicas, Diag. 113 y 64 (s/n), Sucursal 4, CC 16, 1900 La Plata, Argentina

6 Department of Chemistry and Chemical Biology, McMaster University, 1280 Main Street West, Hamilton, ON L8S 4M1, Canada

7 Department of Inorganic and Physical Chemistry, Ghent University, Krijgslaan 281 (S3), 9000 Gent, Belgium

8 Center for Molecular Modeling, Ghent University, Technologiepark 903, 9052 Zwijnaarde, Belgium 
the number of N-electron determinants based on some predetermined criteria $[1,2]$. The best-known CI methods include determinants up to a certain degree of excitation from a reference determinant. By usually strongly reducing the number of determinants the computational cost becomes much more affordable, although by no longer considering the complete space of determinants the energy depends on the number of Slater determinants retained and the orthonormal orbital basis used. Moreover, the coefficients of the $\mathrm{N}$-electron determinants that express the wave functions are basis-set dependent, even in the FCI method. A wave function is considered more compact when fewer determinants have coefficients significantly different from zero. Hence, it is of interest to see whether expanding the CI wave functions in basis sets that render the FCI wave function more compact would result in lower energies in approximate truncated CI wave function. It has long been recognised that much of FCI space consists of deadwood, i.e. configurations that contribute almost nothing to the wave function [1]. This observation inspired work to examine ways to mask off small coefficients in the wave function against a predefined energy threshold, all in the quest of finding an optimally sparse wave function [3, 4]. In the present work, we establish a basis in which the FCI wave function becomes as sparse as possible, i.e. containing as many near-zero coefficients as possible but without altering the FCI energy. The hypothesis is that the basis that results in the most compact FCI wave function may be a good starting point for CI approaches by giving consistently low energies at the truncated levels. It has been commonly accepted that the natural orbitals (NO) [5], which diagonalise the first-order reduced density matrix [6-8], lead to faster expansions than those provided by the canonical Hartree-Fock molecular orbitals (CMO) [9-11]. However, there is no guarantee that the truncated $\mathrm{CI}$ energies arising from the FCI NO basis set are always lower than those obtained from the CMO basis ones. Other types of molecular orbitals have also been proposed, searching for CI expansions with faster convergence than those formulated in terms of natural orbitals. In the present paper we examine the performance of a new basis set that maximises the compactness using entropy considerations. This sets the proposed methods apart from using more localised basis sets [12-15].

We have previously reported results on the compactness of CI and FCI wave functions and their corresponding energies using seniority-number minimising orbitals [16-19]. The seniority number of an $\mathrm{N}$-electron determinant is the number of singly occupied orbitals (or unpaired electrons) in that determinant $[20,21]$. The extension of this concept to $N$-electron wave functions gives unpaired electron number averages for $N$-electron states [22, 23] as the expectation value of the seniority-number operator $[16$,
24]. Because these expectation values are dependent on the basis set utilised to express the wave functions, we have determined orbital sets minimising those expectation values; these resulting orbital sets have been denoted as $M_{\text {min }}$ sets [16]. Our studies have shown that the wave functions expressed in the $M_{\min }$ orbitals are more compact than those based on the CMO and NO basis sets. This more compact nature has been confirmed using the Shannon entropy indices as compactness criterion for expansions of wave functions expressed in the $\mathrm{CMO}, \mathrm{NO}$, and $M_{\min }$ basis sets [25]. However, to our knowledge no study has been made to establish whether there is any connection between the compactness of a FCI wave function and the performance of the underlying basis in truncated CI approaches. Our hypothesis is that a basis that results in a more compact FCI wave function is more biased towards a single determinant and that using this single determinant as a reference would result in lower energies at truncated CI levels. From there, any structure in this basis set could be examined to establish whether one could a priori design better orbital bases for truncated CI. In order to answer this, we describe a new method to specifically minimise the Shannon entropy index and compare the results obtained with those arising from using other basis sets. We also randomly generate a large number of orthonormal bases to examine any trend between truncated CI energies and compactness of the FCI wave function with those bases.

\section{Theoretical aspects}

We express the electronic wave function of an $\mathrm{N}$-electron system according to

$$
\left|\Psi\left(N, S, S_{z}\right)>=\sum_{\Lambda\left(N, S_{z}\right)} C_{\Lambda}^{S}\right| \Lambda\left(N, S_{z}\right)>
$$

in which $S$ and $S_{z}$ are the spin quantum numbers of an $N$-electron spin-adapted wave function $\left|\Psi\left(N, S, S_{z}\right)\right\rangle$. $\mid \Lambda\left(N, S_{z}\right)>$ stands for an $N$-electron Slater determinant, and $C_{\Lambda}^{S}$ is its expansion coefficient. If the summation in Eq. (1) contains all the Slater determinants $\mid \Lambda\left(N, S_{z}\right)>$ that can be constructed with the chosen molecular spin-orbital basis set, expansion (1) corresponds to the FCI wave function. As stated above, limited configuration interaction methods arise from approximating expansion (1), in which some $\mathrm{N}$-electron Slater determinants are neglected according to predetermined criteria, such as the excitation degree of the determinants with respect to a reference determinant [2] or their seniority-number values [16, 24]. An example of the latter criterion is the double-occupied configuration interaction (DOCI) method, in which the $\mid \Psi\left(N, S, S_{z}\right)>$ wave function is expanded in terms of all seniority-zero determinants [26]. 
The spin-free version of the $N$-electron seniority-number operator, $\hat{\Omega}$, is given as [16]

$\hat{\Omega}=\hat{N}-\sum_{i} \hat{E}_{i i}^{i i}$

where $i$ represents the orbitals of a finite orthonormal basis set, $\hat{N}$ is the electron number operator, and $\hat{E}_{i i}^{i i}=\sum_{\sigma_{1}, \sigma_{2}} a_{i^{\sigma_{1}}}^{\dagger} a_{i \sigma_{2}}^{\dagger} a_{i} \sigma_{2} a_{i \sigma_{1}}$ is the second-order replacement operator [27-30] corresponding to the double occupation of the orbital $i$. $a_{i^{\sigma_{1}}}^{\dagger}$ and $a_{i^{\sigma_{1}}}$ are the usual creation and annihilation operators of the spin-orbital $i^{\sigma_{1}}$, respectively, and $\sigma_{1}$ and $\sigma_{2}$ the spin functions. The expectation value of the seniority-number operator $\langle\hat{\Omega}\rangle_{\Psi}=\left\langle\Psi\left(N, S, S_{z}\right)|\hat{\Omega}| \Psi\left(N, S, S_{z}\right)\right\rangle$ is then a weighted sum of the seniority numbers of all determinants involved in expansion (1) [16]. In previous work, we have performed unitary transformations of the molecular orbital set to minimise $\langle\hat{\Omega}\rangle_{\Psi}[16-18,31]$, giving rise to the so-called $M_{\text {min }}$ basis set. This has been done using a specific adaptation of the procedure reported by Subotnik et al. [32].

The invariance of the FCI energy with respect to the orthonormal basis is no longer maintained in the case of limited CI methods. The $C_{\Lambda}^{S}$ expansion coefficients in Eq. (1) depend on the basis set used in both limited CI and FCI procedures. Hence, it is useful to search for molecular basis sets in which the expansion (1) turns out to be more compact also making limited CI series converge more rapidly to the FCI wave functions. This obviously hinges on the presence of an underlying (yet hidden) structure as truncated CI schemes rely on connected one-electron excitations.

One of the quantitative procedures to measure the compactness of a given $N$-electron wave function expressed by Eq. (1) is the informational content $\left(I_{\mathrm{C}}\right)$ index, defined as

$I_{\mathrm{C}}=-\sum_{\Lambda\left(S_{z}\right)}\left|C_{\Lambda}^{S}\right|^{2} \log _{2}\left|C_{\Lambda}^{S}\right|^{2}, \quad\left|C_{\Lambda}^{S}\right| \geq 0$

This and other related indices, which arise from the Shannon information entropy theory [33, 34], have been previously satisfactorily used as a measure of the compactness of a given wave function expressed in several molecular basis sets. These indices have been used both in the traditional excitation-based CI scheme [35] and in the senioritynumber-based one [25]. As mentioned in the Introduction, one of the basis sets sought is that which minimises the $I_{\mathrm{C}}$ value for a given $N$-electron wave function. This scheme associates minimal entropy values with maximal compactness of wave functions. Since it is assumed that the wave function $\mid \Psi\left(N, S, S_{z}\right)>$ is normalised to unity and the coefficients lie in the range $0 \leq\left|C_{\Lambda}^{S}\right| \leq 1$, the entropic index satisfies $I_{\mathrm{C}} \geq 0$. Consequently, compact wave functions, which require as few as possible but consequently high $\left|C_{\Lambda}^{S}\right|$ values, provide low values for $I_{\mathrm{C}}$. The idea behing the use of entropic indices is based on the supposed link between correlation energy and entropy [36-40]. This idea underlies several recent studies, including that by Delle Site that also uses Shannon entropic indices albeit directly on the electron density [41-43].

For minimising the $I_{\mathrm{C}}$ index, we have used the simulated annealing (SA) technique [44, 45]. This has proven to be a very efficient method to find the global minimum of a function possessing many local minima [31]. The SA method consists in emulating a physical process in which a solid system is gradually cooled and frozen in a minimum energy configuration. In the present work, sequences of elementary Jacobi rotations [46] between pairs of orbitals are performed over a randomly chosen angle. The rotation angles are drawn from the normal distribution around zero and limited to a determined interval. Each rotation provides a new $I_{C}$ value. The actual rotation is effectively retained when this value is lower than the previous one; otherwise, a uniform random intermediate parameter is calculated and according to its magnitude the proposed rotation is accepted or rejected. This procedure allows one to escape from local minima. Full technical details of the method can be found in Ref. [31]. In the $I_{\mathrm{C}}$ minimisation set-up, we have both used a scheme where only rotations among orbitals of the same symmetry are considered $I_{\mathrm{C}}(\mathrm{sym})$ and one where the symmetry may be broken $I_{\mathrm{C}}$ (nonsym).

In practice, obtaining a $I_{\mathrm{C}}$ value from FCI is not an efficient approach. However, the present paper first establishes whether a good correlation exists between limited CI energies and $I_{\mathrm{C}}$. In case a meaningful correlation is found, new approaches will be sought to obtain low entropy bases, e.g. by using $I_{\mathrm{C}}$ optimised bases from limited CI calculations.

\section{Computational details}

We have chosen several two-electron systems as well as the beryllium atom, the lithium dimer, and neutral and ionic hydrides of the second-row elements as prototype of systems with four, six, eight and ten electrons, for which the FCI calculations are computationally affordable. In all cases, we have considered the singlet ground state wave functions of these systems, which have been expressed in all the above-mentioned orthonormal orbital bases obtained from the STO-3G atomic basis set, except for the twoelectron systems where larger basis sets have been used. The use of a minimal basis set allows FCI calculations, including the optimisation of $I_{\mathrm{C}}$. Although larger basis sets are required to account more thoroughly for dynamic correlation, in this proof of principle test failure to find a good correlation between limited CI energies and $I_{\mathrm{C}}$ values would indicate that no good correlation can be expected for larger basis sets. The molecular systems have been studied at both equilibrium $\left(R_{\mathrm{e}}\right)$ and symmetrically stretched $\left(R_{\mathrm{st}}\right)$ 
Table 1 Calculated $I_{\mathrm{C}}$ values for the FCI ground state wave functions of selected twoelectron systems expressed in several orthonormal bases using different atomic basis sets

\begin{tabular}{lllllll}
\hline System & Basis & CMO & NO & $M_{\min }$ & $I_{\mathrm{C}}(\mathrm{sym})$ & $I_{\mathrm{C}}($ nonsym $)$ \\
\hline $\mathrm{He}$ & 6-31G & 0.040 & 0.040 & 0.040 & 0.040 & 0.040 \\
& 6-311G & 0.045 & 0.040 & 0.040 & 0.040 & 0.040 \\
$\mathrm{H}_{2}\left(R_{\mathrm{e}}\right)$ & 6-311G(d,p) & 0.084 & 0.079 & 0.079 & 0.079 & 0.079 \\
& 6-31G & 0.141 & 0.119 & 0.119 & 0.119 & 0.119 \\
& 6-311G & 0.158 & 0.124 & 0.124 & 0.124 & 0.124 \\
$\mathrm{H}_{2}\left(R_{\text {st }}\right)$ & 6-311G(d,p) & 0.185 & 0.150 & 0.150 & 0.150 & 0.150 \\
& 6-31G & 0.710 & 0.622 & 0.622 & 0.622 & 0.622 \\
& 6-311G & 0.820 & 0.621 & 0.621 & 0.621 & 0.621 \\
$\mathrm{HeH}^{+}\left(R_{\mathrm{e}}\right)$ & 6-311G(d,p) & 0.810 & 0.608 & 0.608 & 0.608 & 0.608 \\
& 6-31G & 0.087 & 0.067 & 0.067 & 0.067 & 0.067 \\
& 6-311G & 0.088 & 0.063 & 0.063 & 0.063 & 0.063 \\
$\mathrm{HeH}^{+}\left(R_{\mathrm{st}}\right)$ & 6-311G(d,p) & 0.122 & 0.092 & 0.092 & 0.092 & 0.092 \\
& $6-31 \mathrm{G}$ & 0.097 & 0.071 & 0.071 & 0.071 & 0.071 \\
& $6-311 \mathrm{G}$ & 0.097 & 0.068 & 0.068 & 0.068 & 0.068 \\
& $6-311 \mathrm{G}(\mathrm{d}, \mathrm{p})$ & 0.137 & 0.102 & 0.102 & 0.102 & 0.102 \\
\hline
\end{tabular}

Equilibrium distances $\left(R_{\mathrm{e}}\right)$ at experimental or optimised bond lengths and symmetrically stretched ones $\left(R_{\mathrm{st}}\right)$ at $R_{\mathrm{st}}=2.349 R_{\mathrm{e}}\left(\right.$ for $\left.\mathrm{H}_{2}\right), R_{\mathrm{st}}=1.324 R_{\mathrm{e}}\left(\right.$ for $\left.\mathrm{HeH}^{+}\right)$ geometries. The equilibrium distances for the molecules $\mathrm{H}_{2}, \mathrm{HeH}^{+}, \mathrm{LiH}, \mathrm{Li}_{2}, \mathrm{BH}, \mathrm{BeH}_{2}, \mathrm{BH}_{3}, \mathrm{CH}_{4}, \mathrm{NH}_{3}, \mathrm{H}_{2} \mathrm{O}$ and $\mathrm{FH}$ are those reported in Ref. [47]. For $\mathrm{BeH}^{+}$we have used the internuclear distance from Refs. [48, 49], while in $\mathrm{BeH}_{2}^{+}$the geometry was optimised at CI single and double excitation level using the CMO basis set, with the GAUSSIAN code [50]. The one- and two-electron integrals to build the FCI $N$-electron Hamiltonian matrix, as well as the CMO sets, have been obtained from a modified version of the PSI 4 package [51]. Our own codes have been used to calculate the NO, $M_{\min }, I_{\mathrm{C}}(\mathrm{sym})$ and $I_{\mathrm{C}}$ (nonsym) minimising orbital sets as well as a large number of randomly chosen sets, and to perform the required basis-set transformations. The random basis sets were generated by applying unitary matrix transformations on the molecular orbital basis sets. These matrices were built from the eigenvectors of Hermitian matrices. As it was observed that completely random Hermitian matrices resulted systematically in larger values for $I_{\mathrm{C}}$, we included also matrices that are expected to lead to lower $I_{\mathrm{C}}$ values. This was done by stepwise building matrices with an increasing number of orbitals participating in the Jacobi rotations while keeping other orbitals fixed.

\section{Results and discussion}

To allow proper comparison among the different orthonormal basis sets, we first report the $I_{\mathrm{C}}$ values obtained for $\mathrm{CMO}, \mathrm{NO}, M_{\min }$ and $I_{\mathrm{C}}$ minimising sets in both the symmetry and nonsymmetry conserving approaches. Table 1 gathers the $I_{\mathrm{C}}$ values for the two-electron atomic and molecular systems. Table 2 reports the results corresponding to that index for the four- and six-electron systems, while Table 3 collects those values for the eight- and ten-electron ones.

Tables 1, 2 and 3 clearly illustrate the fact that indeed the $I_{\mathrm{C}}$ index value depends on the orthonormal basis chosen for the expansion (1). In case of two-electron systems, Table 1 shows that the values obtained using the NO and $M_{\min }$ bases coincide. This is because a FCI wave function for this type of systems must be expanded in terms of Slater determinants of seniority numbers $\Omega=0$ (with only doubly occupied orbitals) and $\Omega=2$ (with nonrepeated orbitals). However, a unitary transformation allows one to formulate these wave functions as [24, 52, 53]

$\left|\Psi(N=2, S=0)>=\sum_{i} C_{i}\right| i^{\alpha} i^{\beta}>$

in which all the Slater determinants possess seniority number $\Omega=0$. Hence, the value of the $\langle\hat{\Omega}\rangle_{\psi}$ quantity for wave functions of that type is zero, which is the minimum expectation value of the seniority number operator. On the other hand, the spin-free first-order reduced density matrix corresponding to a wave function satisfying Eq. (4) is diagonal and consequently the orbitals $i$ are the natural ones. This argument shows that for two-electron systems in singlet states the NO and $M_{\min }$ basis sets must coincide. The direct minimisation of the $I_{\mathrm{C}}$ index through the simulated annealing technique (in both symmetry and nonsymmetry conserving approaches) also yields that same basis, in accordance with the results reported in Ref. [52].

The results in Table 2 show that all the orbital basis sets considered, except the CMO one, yield identical $I_{\mathrm{C}}$ values in the four-electron systems $\left(\mathrm{Be}, \operatorname{LiH}\left(R_{\mathrm{e}}\right), \mathrm{LiH}\left(R_{\mathrm{st}}\right)\right.$, $\mathrm{BeH}^{+}\left(R_{\mathrm{e}}\right)$ and $\left.\mathrm{BeH}^{+}\left(R_{\mathrm{st}}\right)\right)$. The higher values exhibited by 
the CMO set indicate, as expected, a less compact wave function in that basis. The stretched configurations of the systems $\operatorname{LiH}\left(R_{\mathrm{st}}\right)$ and $\mathrm{BeH}^{+}\left(R_{\mathrm{st}}\right)$ also show systematically higher values with respect to their equilibrium counterparts $\mathrm{LiH}\left(R_{\mathrm{e}}\right)$ and $\mathrm{BeH}^{+}\left(R_{\mathrm{e}}\right)$. These results are coherent with the higher static correlation [24] in the stretched geometries, where the FCI expansion requires a higher number of Slater determinants with significant weight. Table 2 also shows $I_{\mathrm{C}}$ values for the six-electron systems $\left(\mathrm{Li}_{2}\left(R_{\mathrm{st}}\right), \mathrm{BH}\left(R_{\mathrm{e}}\right), \mathrm{BH}\left(R_{\mathrm{st}}\right), \mathrm{BH}_{2}^{+}\left(R_{\mathrm{e}}\right), \mathrm{BH}_{2}^{+}\left(R_{\mathrm{st}}\right), \mathrm{BeH}_{2}\left(R_{\mathrm{e}}\right)\right.$, and $\left.\mathrm{BeH}_{2}\left(R_{\mathrm{st}}\right)\right)$. As can be observed, the highest values of the entropic index are again found with the CMO basis. Only small differences appear between the results with the other bases. The NO and $M_{\min }$ basis sets lead to very similar values, although in the $\mathrm{BH}\left(R_{\mathrm{st}}\right)$ system the $M_{\text {min }}$ basis set provides a markedly more compact wave function than the NO one. The results from the $I_{\mathrm{C}}(\mathrm{sym})$ and $I_{\mathrm{C}}$ (nonsym) optimisations reflect the highest compactness, particularly in the latter case, although the values remain similar to those obtained with the $M_{\text {min }}$ basis. The stretched configurations of these systems also entail very high $I_{\mathrm{C}}$ values with respect to the equilibrium geometries, again coherent with their higher static correlation.

Table 3 describes the results for the eight-electron systems, $\mathrm{BH}_{3}\left(R_{\mathrm{e}}\right)$ and $\mathrm{BH}_{3}\left(R_{\mathrm{st}}\right)$, in which the lowest value of the $I_{\mathrm{C}}$ index is obtained with the $I_{\mathrm{C}}$ (nonsym) optimisation although the $M_{\min }$ basis leads to very similar results. For the ten-electron systems, again the basis sets obtained from the $I_{\mathrm{C}}$ (nonsym) and $M_{\min }$ procedures yield similar results. We do notice that in several cases, releasing the constraints of the $I_{\mathrm{C}}(\mathrm{sym})$ procedure to give the $I_{\mathrm{C}}$ (nonsym) results significantly increases the compaction of the wave function. The similarity of the $I_{\mathrm{C}}$ values for the $I_{\mathrm{C}}$ (nonsym) and $M_{\min }$ bases can be explained as follows. According to Eq. (2), the minimisation of the $\langle\hat{\Omega}\rangle_{\Psi}$ quantity requires the maximisation of the $\sum_{i}\left\langle E_{i i}^{i i}\right\rangle_{\Psi}$ one, which implies reaching wave function expansions with larger coefficients for the Slater determinants containing doubly occupied orbitals $i$, which is equivalent to getting more ordered configurations. The numerical values of the $I_{\mathrm{C}}$ index arising from these last two methods are markedly lower than those provided by the NO basis sets and consequently they must be preferred in the task of compacting wave functions.

In truncated single-reference CI methods, one starts with a single determinant wave function and adds excitations of a given order by replacing occupied orbitals by virtual orbitals. This requires a rationale to distinguish between occupied and virtual orbitals or at least some meaningful order among the orbitals. In case of CMOs, the ordering is based on the expectation values of the Fock operator. The reference determinant is built from the 
Table 3 Calculated $I_{\mathrm{C}}$ values for the FCI ground state wave functions of selected eight- and ten-electron systems expressed in several orthonormal bases using STO-3G basis sets

\begin{tabular}{lllllllllll}
\hline & $\mathrm{BH}_{3}\left(R_{\mathrm{e}}\right)$ & $\mathrm{BH}_{3}\left(R_{\mathrm{st}}\right)$ & $\mathrm{CH}_{4}\left(R_{\mathrm{e}}\right)$ & $\mathrm{CH}_{4}\left(R_{\mathrm{st}}\right)$ & $\mathrm{NH}_{3}\left(R_{\mathrm{e}}\right)$ & $\mathrm{NH}_{3}\left(R_{\mathrm{st}}\right)$ & $\mathrm{H}_{2} \mathrm{O}\left(R_{\mathrm{e}}\right)$ & $\mathrm{H} \mathrm{H}_{2} \mathrm{O}\left(R_{\mathrm{st}}\right)$ & $\mathrm{HF}\left(R_{\mathrm{e}}\right)$ & $\mathrm{HF}\left(R_{\mathrm{st}}\right)$ \\
\hline $\mathrm{CMO}$ & 0.358 & 2.803 & 0.495 & 3.850 & 0.462 & 4.788 & 0.281 & 2.442 & 0.132 & 0.860 \\
$\mathrm{NO}$ & 0.357 & 2.792 & 0.515 & 3.822 & 0.443 & 4.142 & 0.261 & 2.362 & 0.117 & 0.829 \\
$M_{\text {min }}$ & 0.312 & 2.599 & 0.417 & 3.239 & 0.383 & 3.660 & 0.234 & 2.031 & 0.117 & 0.829 \\
$I_{\mathrm{C}}($ sym $)$ & 0.331 & 2.619 & 0.495 & 3.812 & 0.408 & 3.889 & 0.260 & 2.362 & 0.117 & 0.829 \\
$I_{\mathrm{C}}($ nonsym $)$ & 0.312 & 2.598 & 0.417 & 3.239 & 0.383 & 3.659 & 0.234 & 2.030 & 0.117 & 0.829 \\
\hline
\end{tabular}

Equilibrium distances $\left(R_{\mathrm{e}}\right)$ at experimental or optimised bond lengths and symmetrically stretched ones $\left(R_{\mathrm{st}}\right)$ at $R_{\mathrm{st}}=1.750 R_{\mathrm{e}}($ for $\mathrm{BH} 3)$, $R_{\mathrm{st}}=1.750 R_{\mathrm{e}}\left(\right.$ for $\left.\mathrm{CH}_{4}\right), R_{\mathrm{st}}=1.894 R_{\mathrm{e}}\left(\right.$ for $\left.\mathrm{NH}_{3}\right), R_{\mathrm{st}}=1.995 R_{\mathrm{e}}\left(\right.$ for $\left.\mathrm{H}_{2} \mathrm{O}\right), R_{\mathrm{st}}=2.00 R_{\mathrm{e}}($ for $\mathrm{HF})$
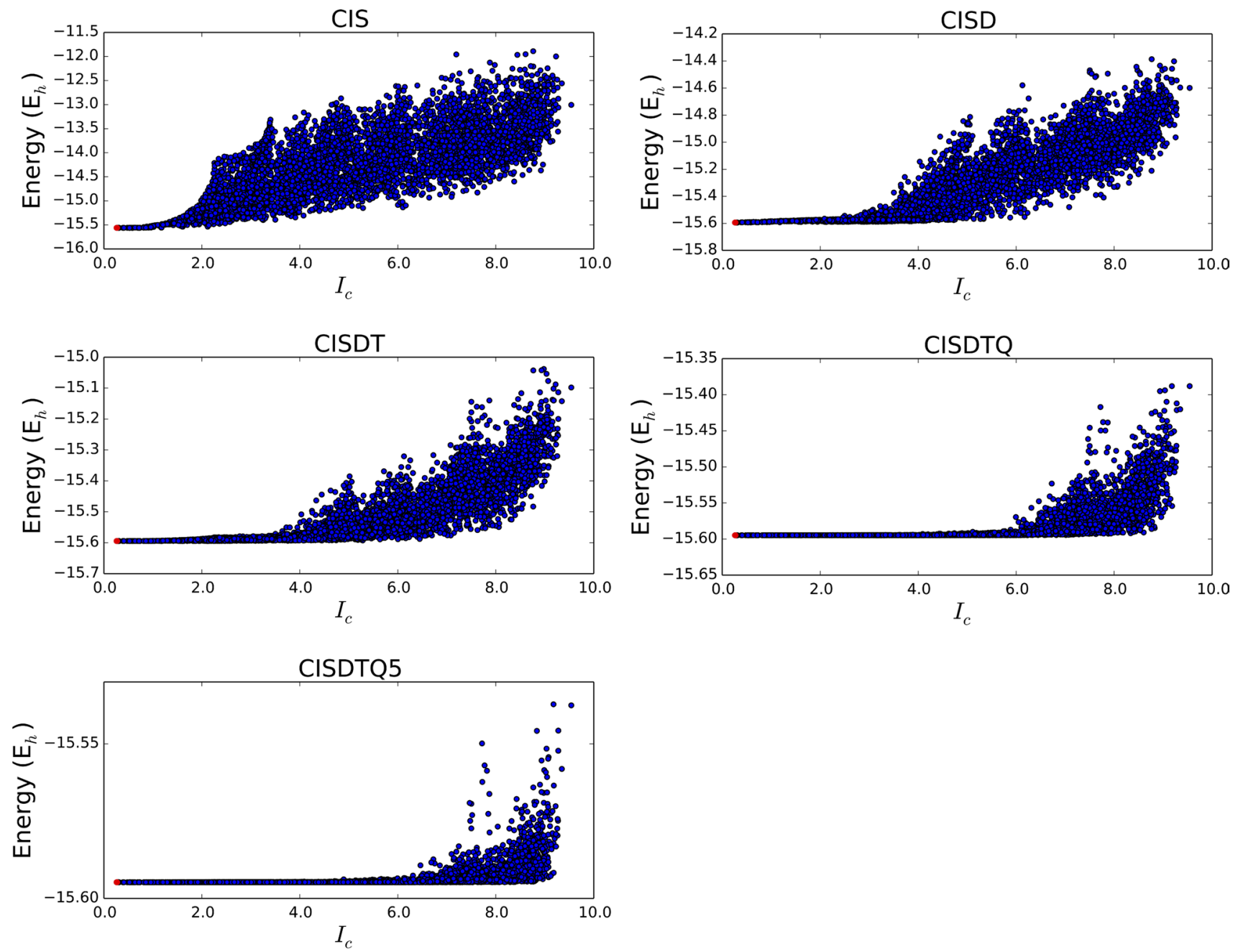

Fig. 1 STO-3G Truncated CI energies for different orthonormal bases versus FCI $I_{\mathrm{C}}$ for $\mathrm{BeH}_{2}$ at equilibrium geometry. The red dot locates the $\mathrm{CMO}, \mathrm{NO}$ and seniority minimising basis sets (indistinguishable on the $I_{\mathrm{C}}$ axis)

lowest energy orbitals. In NOs, one uses the occupation numbers of the natural orbitals and constructs a reference determinant that includes only the most populated natural orbitals. In case of the seniority and $I_{\mathrm{C}}$ minimising orbitals, there appears no simple ordering between the different orbitals in the orthonormal basis. In that case, the most obvious choice is to use as a reference the Slater determinant with the largest absolute coefficient of the FCI wave function.

To examine the possible relationship between the $I_{C}$ value and truncated CI energies, CIS, CISD, CISDT, CISDTQ, and CISDTQ5 energies were computed starting from 

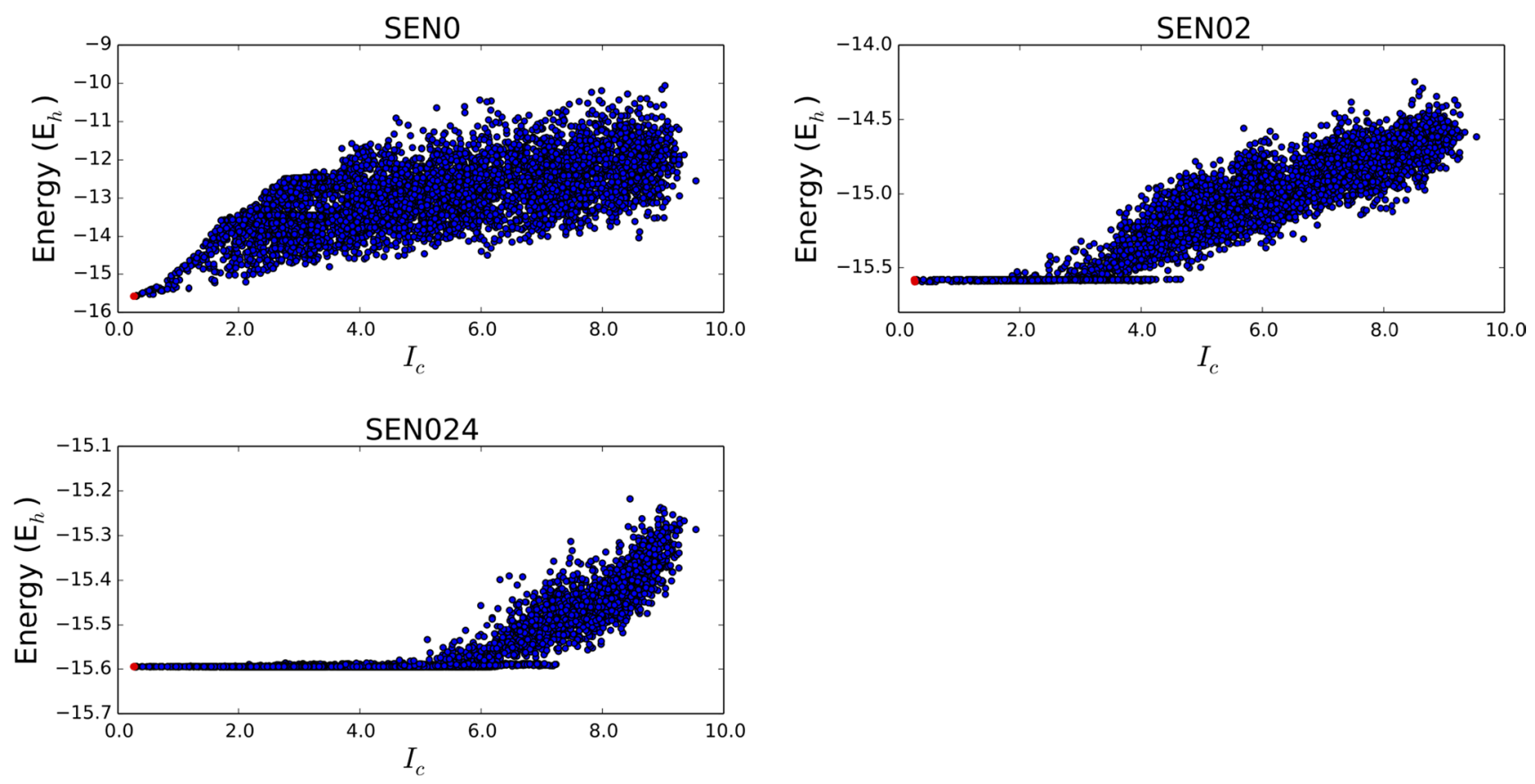

Fig. 2 STO-3G Seniority-based CI energies for different orthonormal bases versus $\mathrm{FCI} I_{\mathrm{C}}$ for $\mathrm{BeH}_{2}$ at equilibrium geometry. The red dot locates the $\mathrm{CMO}, \mathrm{NO}$ and seniority minimising basis sets (indistinguishable on the $I_{\mathrm{C}}$ axis)

the reference determinant obtained from CMOs, NOs, seniority minimising, $I_{\mathrm{C}}$ minimising orbitals and 5000 randomly chosen basis sets obtained as described above. For each basis, the FCI coefficients were computed and from there the $I_{\mathrm{C}}$ values. Figure 1 shows the scatterplot for the truncated $\mathrm{CI}$ energies of $\mathrm{BeH}_{2}$ versus the $I_{\mathrm{C}}$ value computed using the STO-3G basis at the equilibrium geometry. The fact that the CIS energies differ from the Hartree-Fock energy is due to the fact that single excitations generally interact with the reference determinant. Only in case of the Hartree-Fock single determinant, Brillouin's theorem renders these interactions zero. The random basis sets cover the entire range of $I_{\mathrm{C}}$ values from the lowest values obtained with the entropy minimising basis sets to the theoretical upper bound corresponding to equal coefficients for all determinants. As Fig. 1 clearly shows, a qualitative trend may be discerned. However, the main observation is that low truncated CI energies are not exclusively connected to the lowest values of $I_{\mathrm{C}}$. The lowest values of $I_{\mathrm{C}}$ are derived from the $\mathrm{CMO}, \mathrm{NO}$ and seniority and entropy minimising basis sets, but several other random basis sets result in low energies as well although with higher $I_{\mathrm{C}}$ values. This means there is no fundamental quantitative relationship between the $I_{\mathrm{C}}$ values and truncated $\mathrm{CI}$ energies.

The seniority minimising basis results in low entropies (similar to those obtained from the $I_{\mathrm{C}}$ (nonsym) and slightly better than the $I_{\mathrm{C}}(\mathrm{sym})$ basis) and so more compact wave functions and tends to result in low energies for the closed shell systems tested. Besides one-electron excitations, we also explored seniority-based CI expansions such as DOCI (seniority-zero sector), wave functions including the $(0+2)$ sectors (combining DOCI and two unpaired electron determinants), $(0+2+4)$ sector wave functions, and FCI (here equal to $(0+2+4+6)$ wave functions). Figure 2 shows the results, again indicating that there is no obvious relationship. As in Fig. 1, virtually indistinguishable energies are obtained once below a cut-off value in $I_{\mathrm{C}}$. Any fundamental and quantitative relationship between $I_{\mathrm{C}}$ and the truncated CI energies should apply over the entire range of $I_{\mathrm{C}}$ values, including the large number of random basis sets with high $I_{\mathrm{C}}$ in Figs. 1 and 2 and as discussed such a good correlation is absent. As higher excitations are included, more basis sets of quite different $I_{\mathrm{C}}$ values converge to a lower energy plateau as eventually FCI is independent of the basis chosen. That some high $I_{\mathrm{C}}$ basis sets result often in the highest energies is not surprising as one could also imagine maximising the entropy and using one of the many determinants with all (virtually) the same absolute coefficient in the FCI expansion. Still, the main observation is that high entropy does not automatically mean high energy despite an apparent but not quantitative tendency. 
Fig. 3 Symmetry of the orbitals used in this work for the $\mathrm{H}_{2} \mathrm{O}$ at equilibrium geometry. \pm 0.11 a.u. isosurfaces in all plots
$\mathrm{CMO}$

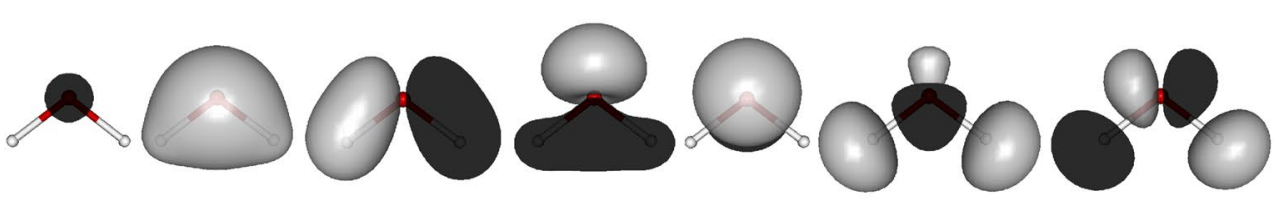

$\mathrm{NO}$
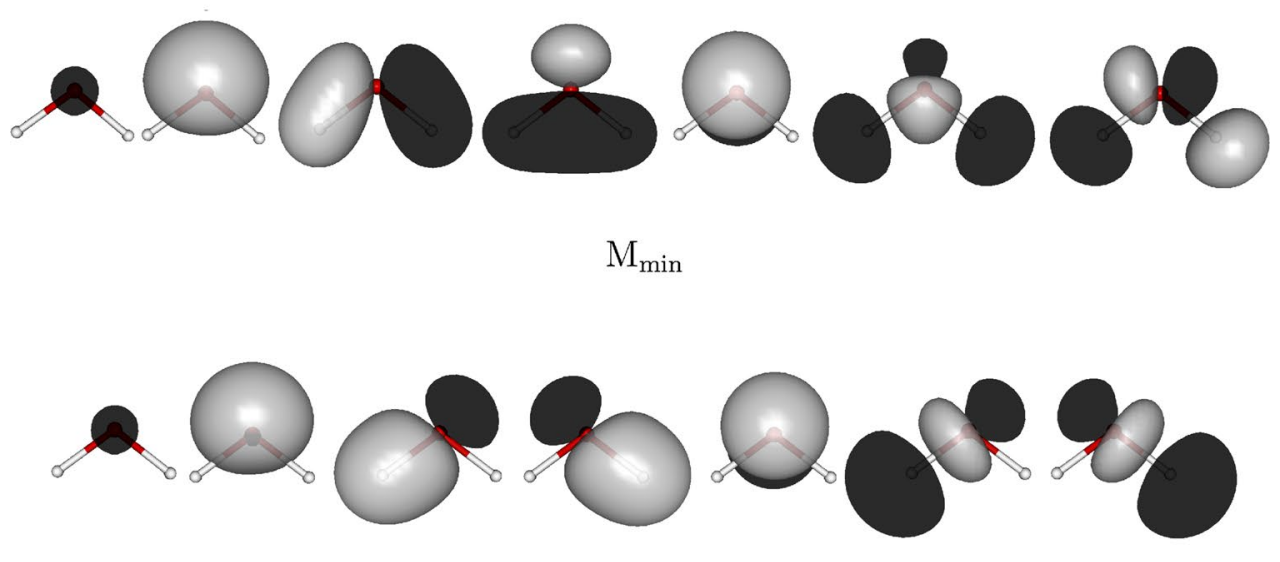

$I_{C}(\mathrm{sym})$
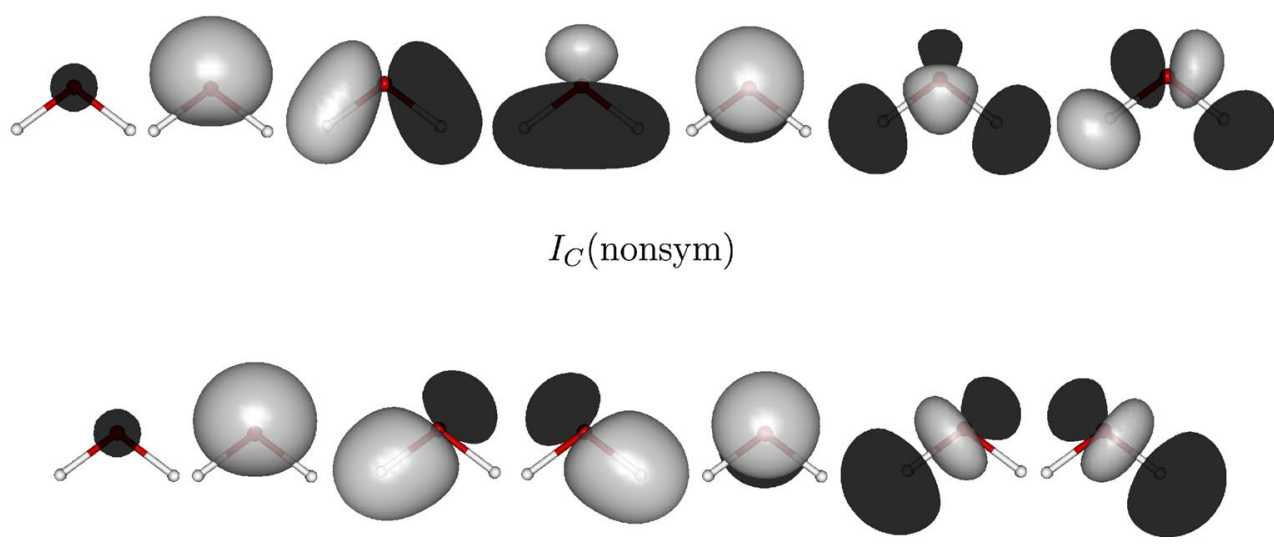

Another aspect mentioned in the Introduction, which deserves to be remarked, is the localisation properties of the orbitals that compose the basis sets used in this work. As is well known, the split-localised natural orbitals are among the best sets in terms of CI series convergence. However, this localisation entails symmetry breaking [14]. We have checked this property plotting in Figs. 3 and 4 the orbitals arising from all reported methods for the $\mathrm{H}_{2} \mathrm{O}$ molecule at the equilibrium and stretched geometries. As can be observed, the $\mathrm{CMO}, \mathrm{NO}$ and $I_{\mathrm{C}}(\mathrm{sym})$ orbitals maintain the symmetry, while it is broken in the cases of the $M_{\min }$ and $I_{\mathrm{C}}$ (nonsym) methods; the unconstrained minimisation of seniority number or entropy causes orbital localisation on atoms and bonding directions, which agrees with genuine chemical behaviour. This agrees with what was previously pointed out in Ref. [14] for natural orbitals (symmetry adapted) and splitlocalised ones where the symmetry adaptation is sacrificed to obtain a better localisation and a faster convergence for CI expansions. 
Fig. 4 Symmetry of the orbitals used in this work for the $\mathrm{H}_{2} \mathrm{O}$ at stretched geometry. \pm 0.11 a.u. isosurfaces in all plots

\section{$\mathrm{CMO}$}

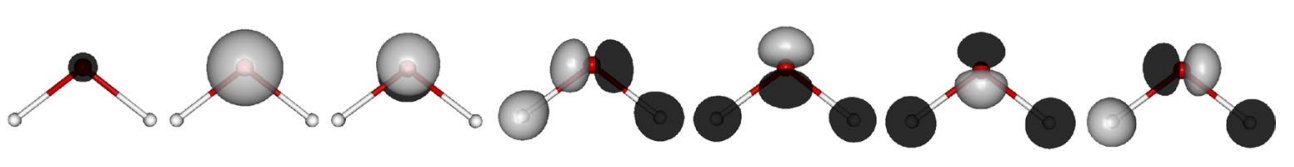

$\mathrm{NO}$
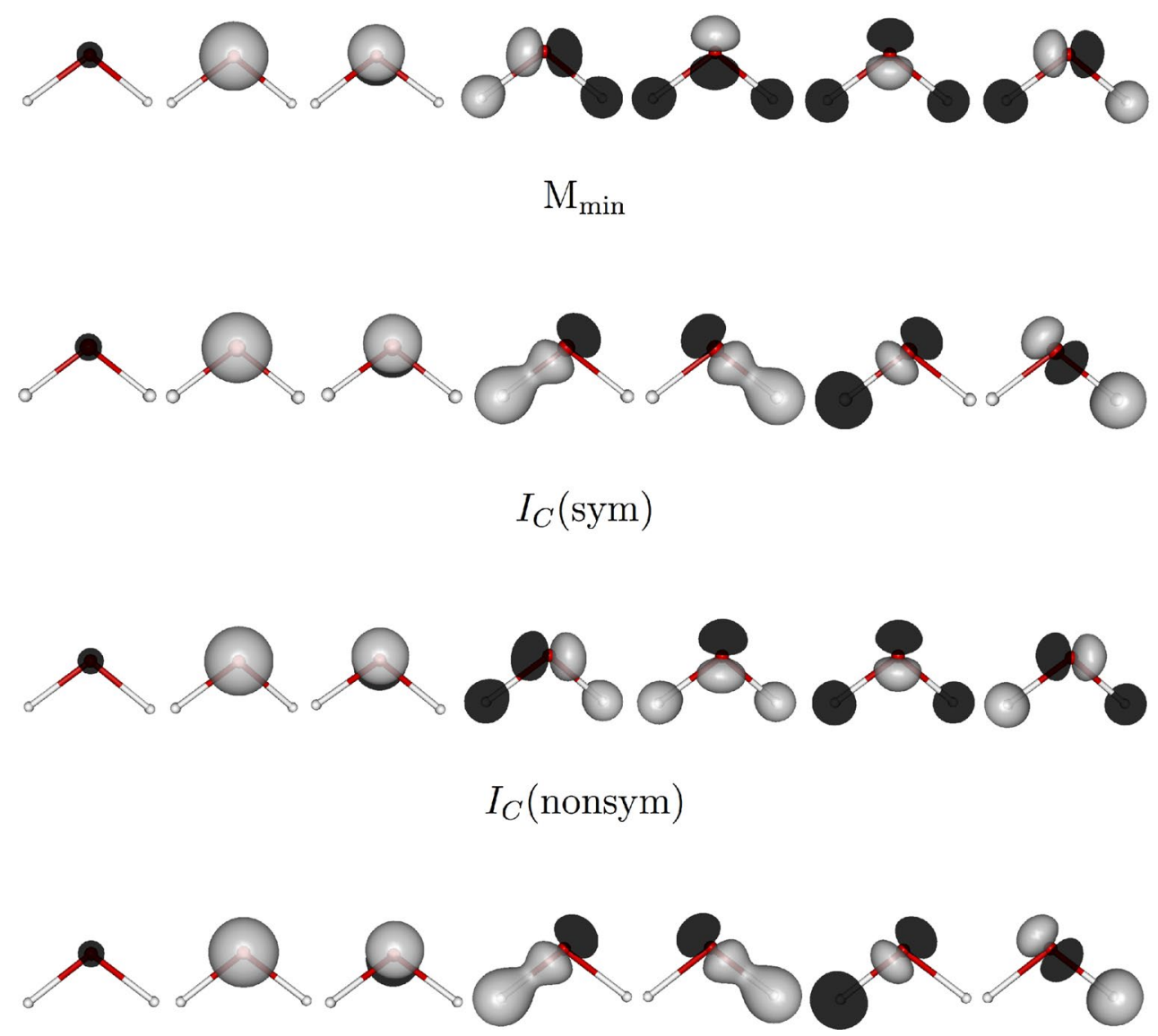

\section{Conclusions}

The compactness of FCI $N$-electron wave functions expressed in different molecular basis sets has been scrutinised, including a basis designed to minimise the Shannon entropy as a benchmark. For the latter, both symmetry conserving and nonsymmetry conserving simulated annealing procedures have been used. Numerical determinations of Shannon entropy indices on several systems using FCI wave functions have revealed that the compactness of the wave functions may depend significantly on the molecular basis.

The seniority-number minimising basis performs nearly as well as the nonsymmetry conserving entropy minimising basis set, and both of them lead to more compact wave functions than the natural orbital and canonical molecular orbital basis sets. In case of two-electron systems, all the studied basis sets, except the canonical molecular orbital one, give rise to the same entropy value due to their special features.

The hypothesis that a more compact FCI wave function would allow us to extract a better single-reference determinant to initiate one-electron excitation-based truncated CI or seniority-based truncated CI calculations that give lower energies is shown to be false. Some basis sets with higher $I_{\mathrm{C}}$ values may still result in low truncated CI energies although there appears to be a cut-off above which 
there is a tendency towards higher energies. Choosing an orbital basis that makes FCI more compact does not seem to automatically lead to more rapidly convergent truncated CI expansions using either the excitation hierarchy or the seniority hierarchy. Choosing the right configurations seems to be more important than choosing the right orbitals.

Acknowledgments D.R.A. and P.B. acknowledge the Ministerio de Ciencia, Tecnología e Innovación Productiva (Argentina) and the FWO Vlaanderen (Belgium) for collaborative research grant VS.0001.14N. D.R.A. acknowledges the Universidad de Buenos Aires (Argentina) and the Consejo Nacional de Investigaciones Científicas y Técnicas (Argentina) for research Grant Nos. UBACYT 20020100100197, PIP 11220090100061 and PIP 11220130100377CO. A.T. and L.L. acknowledge the Universidad del Pais Vasco (Spain) for research Grant Nos. GIU12/09 and UFI11/07. O.B.O. acknowledges the Consejo Nacional de Investigaciones Científicas y Técnicas (Argentina) for research Grant Nos. 11220090100369 and 11220130100311 CO. M.V.R., D.V.N. and P.B. acknowledge the support from the Research Foundation Flanders (FWO Vlaanderen). The computational resources and services used in this work were provided by the Universidad de Buenos Aires and the Universidad del Pais Vasco and the Stevin Supercomputer Infrastructure, provided by the VSC (Flemish Supercomputer Center), funded by Ghent University, the Hercules Foundation and the Flemish Government-department EWI. M.V.R., P.B. and D.V.N. are members of the QCMM alliance Ghent-Brussels.

\section{References}

1. Ivanic J, Ruedenberg K (2001) Identification of deadwood in configuration spaces through general direct configuration interaction. Theor Chem Acc 106(5):339-351

2. Sherill CD, Schaefer HF (1999) The configuration interaction method: advances in highly correlated approaches. Adv Quantum Chem 34:143-269

3. Evangelista FA (2014) Adaptive multiconfigurational wave functions. J Chem Phys 140(12):124114

4. Knowles PJ (2015) Compressive sampling in configuration interaction wavefunctions. Mol Phys 113(13-14):1655-1660

5. Löwdin PO (1955) Quantum theory of many-particle systems.1. Physical interpretations by means of density matrices, natural spin-orbitals, and convergence problems in the method of configurational interaction. Phys Rev 97(6):1474-1489

6. Coleman AJ (1963) Structure of fermion density matrices. Rev Mod Phys 35(3):668-687

7. Bender CF, Davidson ER (1966) A natural orbital based energy calculation for helium hydride and lithium hydride. J Phys Chem 70(8):2675-2685

8. Davidson ER (1976) Reduced density matrices in quantum chemistry. Academic Press, New York

9. Shavitt I (1998) The history and evolution of configuration interaction. Mol Phys 94(1):3-17

10. Kobe DH (1969) Natural orbitals, divergences, and variational principles. J Chem Phys 50(12):5183-5194

11. Shavitt I, Rosenberg BJ, Palalikit S (1976) Comparison of configuration interaction expansions based on different orbital transformations. Int J Quantum Chem Symp 10:33-46

12. Lam B, Schmidt MW, Ruedenberg K (1985) Intraatomic correlation correction in the FORS model. J Phys Chem 89(11):2221-2235
13. Schmidt MW, Gordon MS (1998) The construction and interpretation of MCSCF wavefunctions. Ann Rev Phys Chem 49:233-266

14. Bytautas L, Ivanic J, Ruedenberg K (2003) Split-localized orbitals can yield stronger configuration interaction convergence than natural orbitals. J Chem Phys 119(16):8217-8224

15. Giesbertz KJH (2014) Are natural orbitals useful for generating an efficient expansion of the wave function? Chem Phys Lett 591:220-226

16. Alcoba DR, Torre A, Lain L, Massaccesi GE, Oña OB (2013) Seniority number in spin-adapted spaces and compactness of configuration interaction wave functions. J Chem Phys 139(8):084103

17. Alcoba DR, Torre A, Lain L, Massaccesi GE, Oña OB (2014) Configuration interaction wave functions: A seniority number approach. J Chem Phys 140(23):234103

18. Alcoba DR, Torre A, Lain L, Oña OB, Capuzzi P, Van Raemdonck M, Bultinck P, Van Neck D (2014) A hybrid configuration interaction treatment based on seniority number and excitation schemes. J Chem Phys 141(24):244118

19. Poelmans W, Van Raemdonck M, Verstichel B, De Baerdemacker S, Torre A, Lain L, Massaccesi GE, Alcoba DR, Bultinck P, Van Neck D (2015) Variational optimization of the second-order density matrix corresponding to a seniority-zero configuration interaction wave function. J Chem Theory Comput 11(9):4064-4076

20. Ring P, Schuck P (1980) The nuclear many-body problem. Springer, New York

21. Koltun DS, Eisenberg JM (1988) Quantum mechanics of many degrees of freedom. Wiley, New York

22. Alcoba DR, Bochicchio RC, Lain L, Torre A (2006) On the definition of the effectively unpaired electron density matrix: a similarity measure approach. Chem Phys Lett 429(1-3):286-288

23. Karafiloglou P (2009) An efficient generalized polyelectron population analysis in orbital spaces: the hole-expansion methodology. J Chem Phys 130(16):164103

24. Bytautas L, Henderson TM, Jiménez-Hoyos CA, Ellis JK, Scuseria GE (2011) Seniority and orbital symmetry as tools for establishing a full configuration interaction hierarchy. J Chem Phys 135(4):044119

25. Lain L, Torre A, Alcoba DR, Oña OB, Massaccesi GE (2015) A study of the compactness of wave functions based on shannon entropy indices: a seniority number approach. Theor Chem Acc 134(7):85

26. Weinhold F, Wilson EB (1967) Reduced density matrices of atoms and molecules. I. The 2 matrix of double-occupancy, configuration-interaction wavefunctions for singlet states. J Chem Phys 46(7):2752-2758

27. Paldus J, Jeziorski B (1988) Clifford-algebra and unitary-group formulations of the many-electron problem. Theor Chim Acta 73(2-3):81-103

28. Lain L, Torre A, Karwowski J, Valdemoro C (1988) Matrix-elements of the 3rd-order spin-adapted reduced hamiltonian. Phys Rev A 38(6):2721-2728

29. Torre A, Lain L, Millan J (1993) Calculation of traces of p-order replacement operators over n-electron spin-adapted spaces. Phys Rev A 47(2):923-928

30. Lain L, Torre A (1995) Direct computation of traces of p-order replacement operators over n-electron spin-adapted spaces. Phys Rev A 52(3):2446-2448

31. Van Raemdonck M, Alcoba DR, Poelmans W, De Baerdemacker S, Torre A, Lain L, Massaccesi GE, Van Neck D, Bultinck P (2015) Polynomial scaling approximations and dynamic correlation corrections to doubly occupied configuration interaction wave functions. J Chem Phys 143(10):104106

32. Subotnik JE, Shao YH, Liang WZ, Head-Gordon M (2004) An efficient method for calculating maxima of homogeneous 
functions of orthogonal matrices: Applications to localized occupied orbitals. J Chem Phys 121(19):9220-9229

33. Mathai AM, Tathie PN (1988) Basic concepts in information theory and statistics. Wiley, New York

34. Pfeiffer PE (1978) Concepts of probability theory. Dover, New York

35. Ivanov VV, Lyakh DI, Adamowicz K (2005) New indices for describing the multi-configurational nature of the coupled cluster wave function. Mol Phys 103:2131-2139

36. Collins DM (1993) Entropy maximizations on electron-density. Z Naturforschung A 48(1-2):68-74

37. Esquivel RO, Rodíguez AL, Sagar RP, Hô M, Smith VH (1996) Physical interpretation of information entropy: numerical evidence of the Collins conjecture. Phys Rev A 54(1):259-265

38. Smith GT, Schmider HL, Smith VH (2002) Electron correlation and the eigenvalues of the one-matrix. Phys Rev A 65(3):032508

39. Ziesche P, Smith VH, Hô M, Rudin SP, Gersdorf P, Taut M (1999) The He isoelectronic series and the hooke's law model: correlation measures and modifications of Collins' conjecture. J Chem Phys 110(13):6135-6142

40. Ramírez JC, Soriano C, Esquivel RO, Sagar RP, Hô M, Smith VH (1997) Jaynes information entropy of small molecules: numerical evidence of the Collins conjecture. Phys Rev A 56(6):4477-4482

41. Esquivel RO, López-Rosa S, Dehesa JS (2015) Correlation energy as a measure of non-locality: quantum entanglement of helium-like systems. EPL 111(4):40009

42. López-Rosa S, Esquivel RO, Plastino AR, Dehesa JS (2015) Quantum entanglement of helium-like systems with varying-Z: compact state-of-the-art CI wave functions. J Phys B At Mol Opt Phys 48(17): 175002

43. Delle Site L (2015) Shannon entropy and many-electron correlations: theoretical concepts, numerical results, and Collins conjecture. Int J Quantum Chem 115(19):1396-1404

44. Kirkpatrick S, Gelatt CD, Vecchi MP (1983) Optimization by simulated annealing. Science 220(4598):671-680

45. de Andrade MD, Mundim KC, Malbouisson LAC (2005) Gsa algorithm applied to electronic structure: Hartree-Fock-GSA method. Int J Quantum Chem 103(5):493-499
46. Raffenetti RC, Ruedenberg K, Janssen CL, Schaefer HF (1993) Efficient use of Jacobi rotations for orbital optimization and localization. Theor Chim Acta 86(1-2):149-165

47. Johnson III RD (2006) Nist computational chemistry comparison and benchmark database. URL: http://cccbdb.nist.gov/vibscalejust.asp

48. Roos JB, Larsson M, Larson A, Orel AE (2009) Dissociative recombination of $\mathrm{BeH}^{+}$. Phys Rev A 80(1):012501

49. Chakrabarti K, Tennyson J (2012) Electron collisions with the $\mathrm{BeH}^{+}$molecular ion in the R-matrix approach. Eur Phys J D 66(1):31

50. Frisch MJ, Trucks G, Schlegel H, Scuseria G, Robb M, Cheeseman J, Scalmani G, Barone V, Mennucci B, Petersson G, Nakatsuji $\mathrm{H}$, Caricato M, Li X, Hratchian H, Izmaylov A, Bloino J, Zheng G, Sonnenberg J, Hada M, Ehara M, Toyota K, Fukuda R, Hasegawa J, Ishida M, Nakajima T, Honda Y, Kitao O, Nakai H, Vreven T, Montgomery JJ, Peralta J, Ogliaro F, Bearpark M, Heyd J, Brothers E, Kudin K, Staroverov V, Kobayashi R, Normand J, Raghavachari K, Rendell A, Burant J, Iyengar S, Tomasi J, Cossi M, Rega N, Millam M, Klene M, Knox J, Cross J, Bakken V, Adamo C, Jaramillo J, Gomperts R, Stratmann R, Yazyev O, Austin A, Cammi R, Pomelli C, Ochterski J, Martin R, Morokuma K, Zakrzewski V, Voth G, Salvador P, Dannenberg J, Dapprich S, Daniels A, Farkas O, Foresman J, Ortiz J, Cioslowski J, Fox D (2009) Gaussian, inc., Wallingford CT Gaussian09, revision d.01

51. Turney JM, Simmonett AC, Parrish RM, Hohenstein EG, Evangelista FA, Fermann JT, Mintz BJ, Burns LA, Wilke JJ, Abrams ML, Russ NJ, Leininger ML, Janssen CL, Seidl ET, Allen WD, Schaefer HF, King RA, Valeev EF, Sherrill CD, Crawford TD (2012) PSI4: an open-source ab initio electronic structure program. Wiley Interdiscip Rev Comput Mol Sci 2(4):556-565

52. Löwdin PO, Shull H (1956) Natural orbitals in the quantum theory of 2-electron systems. Phys Rev 101(6):1730-1739

53. Kong L, Valeev EF (2011) A novel interpretation of reduced density matrix and cumulant for electronic structure theories. J Chem Phys 134(21):214109 